\title{
COVID-19 and Neurological Manifestations
}

\author{
Neeraj Balaini ${ }^{1} \quad$ Manish Modi ${ }^{1}$ \\ ${ }^{1}$ Department of Neurology, Postgraduate Institute of Medical \\ Education and Research, Chandigarh, India
}

\begin{abstract}
Address for correspondence Manish Modi, DM, Department of Neurology, Postgraduate Institute of Medical Education and Research, Chandigarh 160012, India (e-mail: modipgi@gmail.com).
\end{abstract}

\begin{abstract}
Keywords

- coronavirus

- COVID-19

- neurologic complications

Coronavirus disease 2019 (COVID-19) is a viral illness caused by severe acute respiratory syndrome coronavirus-2 (SARS-CoV-2) which has taken the form of a pandemic. It mainly presents as fever, cough, shortness of breath involving respiratory system but neurological manifestations are increasingly being recognized worldwide and even virus RNA was demonstrated to be present in cerebrospinal fluid of a patient. SARS-CoV-2 involves both central nervous system and peripheral nervous system. Virus can enter the neural tissue from hematological route or through retrograde transport from nerve endings. Physicians especially neurologists should be aware regarding neurological manifestations as patient can present with these conditions in emergency. We therefore reviewed the neurological diseases or complications associated with COIVID-19 in available literature.
\end{abstract}

\section{Introduction}

The Coronavirus disease 2019 (COVID-19), a viral illness first noticed in Wuhan, China, has taken the form of pandemic. ${ }^{1}$ The International Virus Classification Commission has named this novel Coronavirus as severe acute respiratory syndrome Coronavirus-2 (SARS-CoV-2). It is a positive-sense single-stranded RNA virus having a 88 to $96 \%$ similarity of genetic sequence to the bat Coronavirus; therefore, SARS-CoV-2 is postulated to have originated from bats. Two other epidemics caused by the Coronavirus are severe acute respiratory syndrome (SARS), with around $10 \%$ mortality rate, and MERS (Middle East respiratory syndrome) with $37 \%$ mortality. ${ }^{2}$ But compared with them, SARS-CoV-2 is highly transmissible probably because of higher affinity to angiotensin-converting enzyme-2 (ACE-2) receptor, using which the virus gains entry into the host. ${ }^{3}$ The most common symptoms of COVID-19 pertaining to the respiratory system include fever, cough, shortness of breath and acute respiratory distress syndrome (ARDS), but other systems' involvement is being increasingly recognized as cases are rising. It can involve neurological, renal, cardiac, and circulatory systems. Neurological involvement is seen in 8 to $36 \%$ percent of patients in different studies. ${ }^{4-6}$ Neurological manifestations were reported to be more in severe COVID-19 disease as compared with patients with mild disease. ${ }^{4}$ We present a descriptive review of neurological complications of COVID-19.

\section{Materials and Methods}

We searched PubMed for relevant literature using keywords "Coronavirus," "COVID-19," "manifestations," "neurological," "anosmia" and "ophthalmoparesis." We selected articles only in the English language and included case reports, letters to editor, review articles, and neurological images. We also focused on articles in which the mechanism of neurological insult was also described.

\section{Pathogenesis of Neurological Involvement in COVID-19}

The exact pathogenesis of COVID-19 is yet to be established. Studies on SARS-related Coronavirus (SARS-CoV) are extrapolated because of the similarity between the two viruses. SARS-CoV-2 uses ACE-2 receptor as a host cell receptor. ACE-2 is expressed in brain tissue, although less as compared with small intestine and lungs. ${ }^{7}$ After gaining entry into the body, the virus replicates intracellularly, evades host immune response, and leads to cascade of events, causing increased proinflammatory cytokines IFN- $\gamma$, IFN- $\alpha$, IL-6, IL-12, IL-18, IL-33, TNF- $\alpha$ and chemokines (CXCL9, CCl2, CCL3, CCL5, etc.). Increased proinflammatory cytokines may lead the immune system to attack its own body, causing systemic inflammatory response, ARDS and multiple organ failure. ${ }^{2}$ Once the virus gains entry into the host body, neurological involvement can occur in the following ways (-Fig. 1).
License terms

$10.1055 / \mathrm{s}-0040-1714153$

ISSN 0379-038X. 


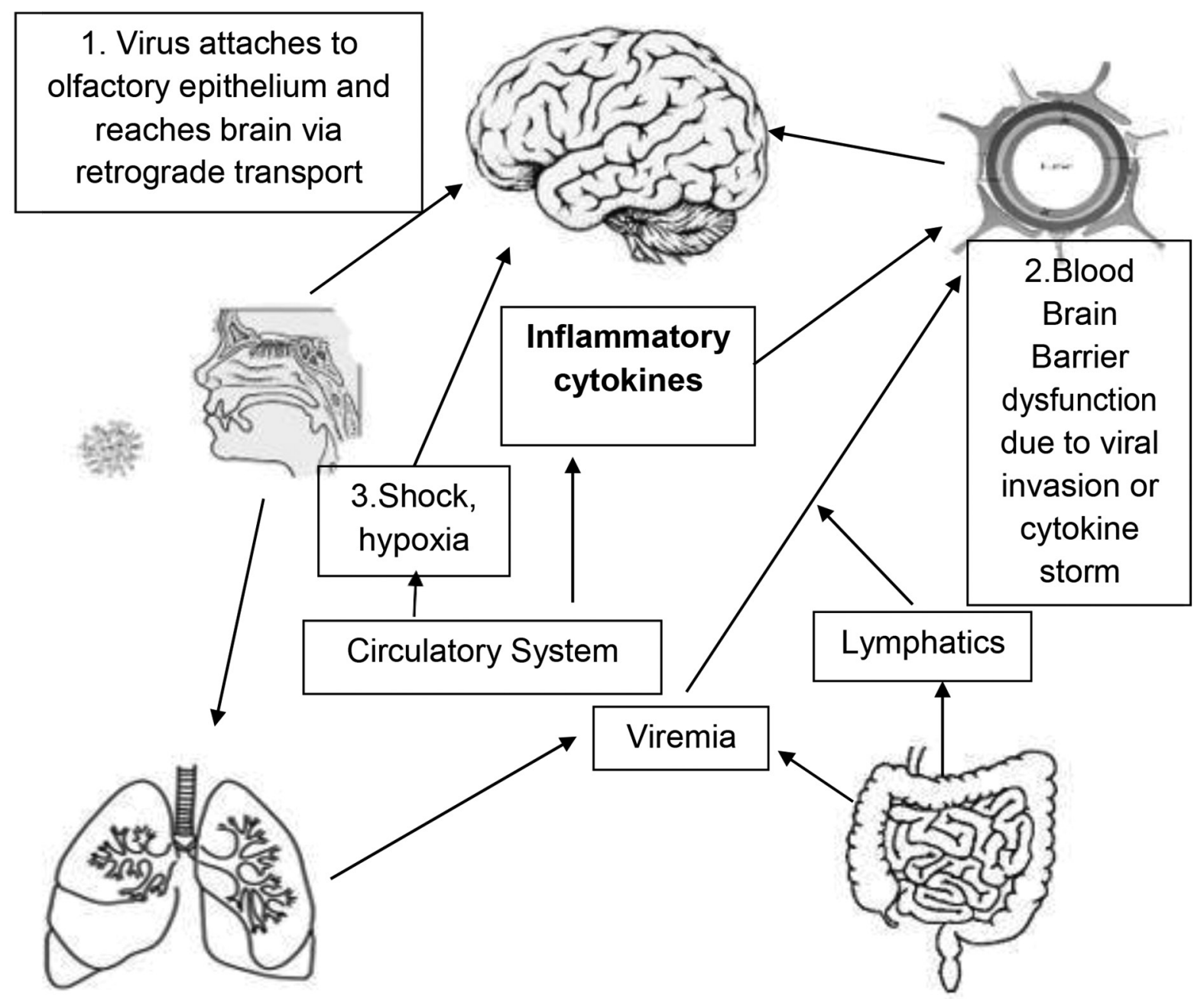

Fig. 1 Mechanism of neurological manifestations in COVID-19: 1. Direct viral invasion via retrograde transport through nerves. 2. Hematogenous dissemination through circulatory system. 3. Secondary to hypoxia, shock, and other systemic complications.

I. Direct invasion of central nervous system (CNS) by virus.

II. Aberrant immune response.

III. Secondary to systemic complications such as hypoxia or shock.

I. Direct invasion by virus into CNS: SARS-CoV-2 can enter the CNS by two routes-following a neural pathway or through body fluids (lymph, blood). The virus may enter into a nerve terminal, multiply, and be retrogradely transported to a cell body of neurons, for example, hyposmia in SARS-CoV-2. The virus first infects the olfactory epithelium and then can be transported into the CNS along the olfactory nerve. Evidence of such direct invasion into CNS comes from studies on other Coronaviruses. Netland et al demonstrated that, SARS-CoV after inoculation through intranasal route in mice that are transgenic for SARS-CoV receptor (human angiotensin-converting enzyme-2), viral antigen was present in olfactory bulb specimens after 60 to 66 hours and subsequently involved piriform and infralimbic cortex, basal ganglia and midbrain without demonstrating viral antigen in intestine or lung tissue, suggesting direct spread from olfactory epithelium and olfactory nerve to brain tissue. ${ }^{8} \mathrm{~A}$ similar study by Dube et al in mice inoculated with human Coronavirus (HCOV-OC43) demonstrated spread from olfactory neuroepithelium to brain. ${ }^{9}$ Furthermore, the virus can gain direct entry into brain via the hematogenous route. After gaining entry through lungs or intestine. the virus particles can directly enter the blood stream or infect local lymph nodes, that is, hilar and mesenteric lymph nodes, and eventually into the blood circulation through lymphatics. ${ }^{3}$ The virus then infects endothelial cells of the blood-brain barrier (BBB) and subsequently spreads across the CNS. The BBB can also be disrupted by a cytokine storm followed by CNS invasion. ${ }^{10}$

II. Aberrant immune response: As pointed above, SARS$\mathrm{CoV}-2$ infection triggers aberrant immune response which can lead to CNS manifestations due to immune mechanisms targeting CNS or peripheral nervous system (PNS), ${ }^{11}$ for example, Guillain-Barre Syndrome (GBS) or cranial neuropathies in SARS-CoV-2.

III.Secondary to systemic involvement: SARS-CoV-2 can cause multiple organ failure and CNS involvement can be secondary to systemic involvement, for example, hypoxic encephalopathy, uremic encephalopathy, intracranial 
bleed or thrombosis due to disseminated intravascular coagulopathy (DIC).

\section{Neurological Involvement in COVID-19}

Neurological involvement could mean CNS or PNS involvement (-Table 1).

A. Central nervous system manifestations: CNS manifestations can be divided into meningeal involvement, brain parenchymal (focal or diffuse) involvement, vascular involvement, spinal cord manifestations, and other nonspecific CNS involvement.

A.1. Meningeal involvement: Meningeal involvement causes headache, meningitis or meningoencephalitis (meningeal and brain parenchymal involvement). Headache incidence reported in literature ranges from 8 to $13.6 \%$.,5,12 Headache is nonspecific and its pathophysiology has not been described in these studies. Although one case report described a COVID-19 patient who presented with meningoencephalitis had headache and SARS-CoV-2 RNA was detected in his CSF. ${ }^{13}$ This points toward the possibility that headache can be due to meningeal involvement or inflammation due to virus spread, leading to a mild form of aseptic meningitis. Two cases of meningoencephalitis have been reported due to COVID-19, one each from Japan and Wuhan (China). ${ }^{13,14}$

A.2. Brain parenchymal involvement (focal or diffuse): Brain parenchymal involvement due to COVID-19 documented to date presented differently as encephalitis (meningoencephalitis), seizures, ataxia, and acute hemorrhagic necrotizing encephalopathy. Seizures can occur secondary to meningoencephalitis. The reported incidence of seizures in COVID-19 is $0.5 \%$ in a study of 214 patients from Wuhan. ${ }^{4}$ In the same study, ataxia was seen in $0.5 \%$ patients, although the mechanism for seizure and ataxia was not discussed. Acute hemorrhagic necrotizing encephalopathy has been reported in a female patient of COVID-19 in her late fifties. The mechanism postulated is an intracranial cytokine storm, leading to BBB disruption. ${ }^{15}$ Helms et al reported a study from France where out of 64 patients admitted with ARDS due to COVID-19, 69\% had agitation on discontinuing neuromuscular blockade, $67 \%$ patients were having diffuse corticospinal signs, and 33\% patients were having poor executive functions at discharge. Brain MRI of 13 patients was done for unexplained encephalopathy in this study, and it showed leptomeningeal enhancement in 8 patients and subclinical infarct in two patients; also, 11 patients were having bilateral frontotemporal hypoperfusion in perfusion studies. ${ }^{16}$

A.3. Vascular involvement: Acute cerebrovascular disease in the form of ischemic stroke or hemorrhagic stroke and cortical venous thrombosis has been reported in COVID-19 patients. ${ }^{17}$ Mao et al reported stroke in six patients (2.8\%) out of 214 hospitalized COVID-19 patients in Wuhan, with five suffering an ischemic stroke and one suffering a hemorrhagic stroke. Stroke was even more with severe COVID-19 illness, according to the study. ${ }^{4}$ Cortical venous thrombosis has also been reported in COVID-19 patients. ${ }^{17}$

Table 1 Neurological complications of COVID-19

\begin{tabular}{|c|c|c|c|}
\hline \multirow[t]{12}{*}{1} & \multirow[t]{12}{*}{ CNS involvement } & \multirow[t]{3}{*}{ Meningeal involvement } & Headache \\
\hline & & & Meningitis \\
\hline & & & Meningoencephalitis \\
\hline & & \multirow{4}{*}{$\begin{array}{l}\text { Brain parenchymal involvement } \\
\text { (focal or diffuse) }\end{array}$} & Encephalitis \\
\hline & & & Seizures/epilepsy \\
\hline & & & Ataxia \\
\hline & & & Acute necrotizing encephalopathy \\
\hline & & \multirow[t]{3}{*}{ Vascular involvement } & Ischemic stroke \\
\hline & & & Hemorrhagic stroke \\
\hline & & & Cerebral venous thrombosis \\
\hline & & Spinal cord & Transverse myelitis \\
\hline & & $\begin{array}{l}\text { Secondary to systemic } \\
\text { complications }\end{array}$ & Encephalopathy Headache Dizziness \\
\hline \multirow[t]{7}{*}{2} & \multirow[t]{7}{*}{ PNS involvement } & \multirow[t]{5}{*}{ Cranial neuropathies } & Hyposmia/anosmia \\
\hline & & & Hypogeusia/Ageusia \\
\hline & & & Chemesthesis Loss/impairment \\
\hline & & & Ophthalmoparesis \\
\hline & & & Vision loss \\
\hline & & Peripheral nerves & GBS and variants (MFS) \\
\hline & & Skeletal muscle & Myalgia/raised creatine kinase \\
\hline
\end{tabular}

Abbreviation: PNS, peripheral nervous system. 
The mechanism of stroke is not well-elucidated in COVID-19 but extrapolating findings from other organs can provide explanations. Autopsy studies demonstrated deep venous thrombosis in lower limbs, and pulmonary thromboembolism and microthrombi in small lung arteries and within the prostate. ${ }^{18}$ Endothelitis was seen in kidney and small intestine vessels on electron microscopy. ${ }^{19}$ Coagulopathy in COVID-19 is combination of DIC and thrombotic microangiopathy with thrombocytopenia, elevated D-dimer, and prolonged prothrombin time along with endothelial dysfunction. Thrombocytopenia in COVID-19 is not as severe as sepsis but D-dimer is more profoundly elevated. Proinflammatory cytokines may have to play a role in increasing the coagulability of blood as well as in endothelial dysfunction. ${ }^{20}$ Direct viral invasion into endothelium also causes endothelial dysfunction. Similar mechanisms in brain can lead to ischemic as well as hemorrhagic stroke.

A.4. Spinal cord: Transverse myelitis has been reported in one COVID-19 patient.

A 66-year-old SARS-CoV-2 positive male developed acute transverse myelitis on the 7th day of illness, which was reported from Wuhan, China. He improved with IV immunoglobulin. It was attributed to inflammation due to a cytokine storm. ${ }^{21}$

A.5. Nonspecific/secondary to systemic involvement: Headache, nausea, vomiting, dizziness, encephalopathy, and delirium have been reported. Dizziness is a nonspecific symptom present in 8 to $16 \%$ patients with COVID-19 in two studies. ${ }^{4,6}$ However, dizziness was not further explored in these studies nor was the mechanism of dizziness elucidated. Nausea and vomiting also have been observed in COVID-19 patients, which can be attributed either to gastrointestinal involvement or area postrema/chemoreceptor trigger zone CTZ involvement in brain. Encephalopathy can be secondary to hypoxia, uremia or other systemic complications and not specific for CNS involvement. Delirium also can be multifactorial in COVID-19 patients.

B. PNS manifestations: PNS manifestations reported to date are in the form of cranial neuropathies, peripheral nerve involvement in the form of GBS and variants, and skeletal muscle involvement.

B.1. Cranial neuropathies: Among cranial neuropathies, hyposmia and hypogeusia are common. Oral chemesthesis loss, ophthalmoparesis, and polyneuritis cranialis have also been documented. Olfactory dysfunction and taste dysfunction are being increasingly recognized as early symptoms of COVID-19. Incidence of olfactory dysfunction reported from different studies ranges between 5.1 to $85.6 \%$ and taste dysfunction incidence ranges between 5.6 to $88 \%{ }^{4,22}$ In fact, olfactory dysfunction was the first symptom in 11.8 to $26.6 \%$. Olfactory dysfunction documented in studies are anosmia, hyposmia, parosmia, and phantosmia in descending order, according to the number of cases. ${ }^{22}$ Oral chemesthesis impairment has also been described with COVID-19.

Chemesthesis means to sense and detect chemical environment around us in daily life. This sensation is different from taste and olfaction, for example, spices evoking sensations like tingling, cooling or burning, and menthol from mint, producing a cooling sensation. Parma et al reported $46 \%$ rating reduction for chemesthesis accompanied by either taste or smell loss in 4039 participants. ${ }^{23}$ This suggests that SARS-CoV-2 affects all three chemosensory modalities-taste, olfaction and chemesthesis. Multiple hypotheses have been postulated for taste and olfactory dysfunction. 1) Local inflammatory response: SARS-CoV-2 triggers a local inflammation, causing rhinitis or stomatitis which can cause dysfunction of olfactory epithelium and taste receptors. 2) Local immune response: virus triggers a local immune response, leading to antibody formation against components of olfactory and gustatory tissues. 3) Cytopathic effect: SARS-CoV-2 virus interacts with ACE- 2 receptors over olfactory and gustatory cells and causes dysfunction of these modalities due to the neurocytopathic effect. 4) Altered neurotransmission in olfactory and gustatory pathways, affecting serotonin and dopamine pathways. 5) Central involvement of olfactory bulb and other brain structures can lead to taste and olfactory sense dysfunction. ${ }^{24}$ The exact mechanism causing chemesthesis impairment is not known but similar mechanisms may be operational. Vision involvement was present in $1.4 \%$ patients out of 214 patients in a study from Wuhan, China. ${ }^{4}$ However, the type of visual involvement is not described further. Two patients were reported, one each from Madrid, Spain, and USA with ophthalmoparesis in COVID-19 patients due to bilateral abducens nerve palsy in one patient and unilateral right abducens nerve palsy in the other patient, respectively. MRI showed optic nerve sheath enhancement and posterior tenon capsule enhancement in the second patient. ${ }^{11,25}$

B.2. Peripheral nerve involvement: GBS in patients with COVID-19 have been reported from Italy, China and Iran. ${ }^{26-28}$ Five patients of COVID-19 developed GBS after 5 to 10 days of illness. Three patients had an axonal variant of GBS, and two patients had a demyelinating variant. They were treated with IV immunoglobulin for GBS. GBS variants were also associated with COVID-19. Miller Fisher Syndrome (MFS) was reported in two patients, one each from Italy and USA. ${ }^{9,22}$ GD1b antibody was positive in the patient from Italy and both were treated with IV immunoglobulin. Aberrant immune response was the proposed mechanism described in these studies pertaining to the development of GBS and MFS. COVID-19 patients in critical care can also develop critical illness-associated neuropathy or myopathy although they are not directly related to COVID-19 infection.

B.3. Skeletal muscle involvement: Myalgia is a common complaint in COVID-19 patients ranging from 14.9 to $44 \%$, although some studies have taken myalgia and fatigue together in their analysis.,6 Skeletal muscle involvement in the form of myalgia and raised creatine kinase more than $200 \mathrm{U} / \mathrm{L}$ was found in $10.7 \%$ patients out of 214 patients in Wuhan by Mao et al. ${ }^{4}$ Proposed mechanism for muscle injury is a direct effect of COVID-19 virus on muscle or proinflammatory cytokines, causing skeletal muscle injury. 


\section{Management of Neurological Complications of COVID-19}

Along with standard management of COVID-19, neurological complications may need separate treatment, according to the type of neurological involvement. Minor symptoms such as headache, dizziness and myalgia can be managed symptomatically. Meningitis or meningoencephalitis needs ruling out other causes of similar presentation and empirical treatment with antibiotics and antivirals till etiology is established. Antiepileptics should be given for seizures, according to institutional protocol. Patients presenting as acute ischemic stroke also can be managed as per international/regional acute stroke guidelines. But one should be cautious as coagulopathy and other systemic involvement like uremia, ARDS may be coexisting and may alter the outcome. For complications arising out of aberrant immune response like GBS, MFS, acute necrotizing encephalopathy, intravenous immunoglobulins are treatment of choice. For isolated cranial neuropathies only follow-up may be needed as they tend to resolve spontaneously or steroids or IVIg can be given after individualizing each patient.

\section{Future Research}

Our understanding of COVID-19 is still incomplete and presently the world is busy in dealing with the catastrophe caused by the virus. As the cases are on rise and an increasing amount of research is ongoing, we can see more of neurological manifestations and understand the underlying pathophysiology in a better way. Further research is needed to determine the exact pathway of entry of the virus in the CNS, and potential therapeutic agents which can target the virus before it invades body tissues, activating a cascade of events and leading to a cytokine storm.

\section{Conclusion}

Neurological complications are increasingly being recognized in COVID-19, and these manifestations can be the first presenting features in emergency. Neurologists are required to have a high-index of suspicion in such cases to identify and manage the patient.

\section{Conflict of Interest}

None declared.

\section{References}

1 World Health Organization. Coronavirus Disease 2019 (COVID-19) Situation Report - 111. Available at: https://www. who.int/docs/default-source/coronaviruse/ situation-reports /20200510covid-19-sitrep-111.pdf?sfvrsn=1896976f_2. Accessed June 12, 2020

2 Li X, Geng M, Peng Y, Meng L, Lu S. Molecular immune pathogenesis and diagnosis of COVID-19. J Pharm Anal 2020;10(2):102-108

3 Li Z, Liu T, Yang N, et al. Neurological manifestations of patients with COVID-19: potential routes of SARS-CoV-2 neuroinvasion from the periphery to the brain. Front Med 2020. Doi: 10.1007/ s11684-020-0786-5

4 Mao L, Jin H, Wang M, et al. Neurologic manifestations of hospitalized patients with Coronavirus disease 2019 in Wuhan, China.JAMA Neurol 2020. Doi: 10.1001/jamaneurol.2020.1127

5 Guan WJ, Ni ZY, Hu Y, et al; China Medical Treatment Expert Group for Covid-19. Clinical characteristics of Coronavirus ddisease 2019 in China. N Engl J Med 2020;382(18):1708-1720

6 Chen T, Wu D, Chen H, et al. Clinical characteristics of 113 deceased patients with coronavirus disease 2019: retrospective study. BMJ 2020;368:m1091

7 Li MY, Li L, Zhang Y, Wang XS. Expression of the SARS-CoV-2 cell receptor gene ACE2 in a wide variety of human tissues. Infect Dis Poverty 2020;9(1):45

8 Netland J, Meyerholz DK, Moore S, Cassell M, Perlman S. Severe acute respiratory syndrome coronavirus infection causes neuronal death in the absence of encephalitis in mice transgenic for human ACE2. J Virol 2008;82(15):7264-7275

9 Dubé M, Le Coupanec A, Wong AHM, Rini JM, Desforges M, Talbot PJ. Axonal transport enables neuron-to-neuron propagation of human coronavirus OC43. J Virol 2018;92(17):e00404-e00418

10 Baig AM, Khaleeq A, Ali U, Syeda H. Evidence of the COVID-19 virus targeting the CNS: tissue distribution, host-virus interaction, and proposed neurotropic mechanisms. ACS Chem Neurosci 2020;11(7):995-998

11 Gutiérrez-Ortiz C, Méndez A, Rodrigo-Rey S, et al. Miller Fisher Syndrome and polyneuritis cranialis in COVID-19. Neurology 2020. Doi: 10.1212/WNL.0000000000009619

12 Huang C, Wang Y, Li X, et al. Clinical features of patients infected with 2019 novel coronavirus in Wuhan, China. (published correction appears in Lancet. January 30, 2020) Lancet 2020;395(10223):497-506

13 Moriguchi T, Harii N, Goto J, et al. A first case of meningitis/ encephalitis associated with SARS-Coronavirus-2. Int J Infect Dis 2020;94:55-58

14 Ye M, Ren Y, Lv T. Encephalitis as a clinical manifestation of COVID-19. Brain Behav Immun 2020. Doi: 10.1016/j. bbi.2020.04.017

15 Poyiadji N, Shahun G, Noujaim D, Stone M, Patel, S, Griffith, B, COVID-19 associated acute hemorrhagic necrotizing encephalopathy: CT and MRI features. Radiology; 2020 Doi:10.1148/ radiol.2020201187

16 Helms J, Kremer S, Merdji H, et al. Neurologic features in severe SARS-CoV-2 Infection. Engl J Med 2020;382(23):2268-2270

17 Asadi-Pooya AA, Simani L. Central nervous system manifestations of COVID-19: A systematic review. J Neurol Sci 2020;413(April):116832

18 Wichmann D, Sperhake JP, Lütgehetmann M, et al. Autopsy findings and Venous Thromboembolism in patients with COVID-19. Ann Intern Med 2020. Doi: 10.7326/M20-2003

19 Varga Z, Flammer AJ, Steiger P, et al. Endothelial cell infection and endotheliitis in COVID-19. Lancet 2020;395(10234): 1417-1418

20 Levi M, Thachil J, Iba T, Levy JH. Coagulation abnormalities and thrombosis in patients with COVID-19. Lancet Haematol 2020;7(6):e438-e440

21 Zhao K, Huang J, Dai D, Feng Y, Liu L, Nie S, Acute myelitis after SARS-CoV-2 infection: a case report. MedRxiv 2020. Doi: 10.1101/2020.03.16.20035105

22 Lechien JR, Chiesa-Estomba CM, De Siati DR, et al. Olfactory and gustatory dysfunctions as a clinical presentation of mildto-moderate forms of the coronavirus disease (COVID-19): a multicenter European study. Eur Arch Otorhinolaryngol 2020. Doi: 10.1007/s00405-020-05965-1

23 Parma V, Ohla K, Veldhuizen MG, et al More than just smell-COVID19 is associated with severe impairment 
of smell, taste and chemesthesis. medRxiv 2020. Doi: 10.1101/ 2020.05.04.20090902

24 Finsterer J, Stollberger C. Causes of hypogeusia/hyposmia in SARS-CoV2 infected patients. J Med Virol 2020. Doi: 10.1002/ jmv.25903

25 Dinkin M, Gao V, Kahan J, et al. COVID-19 presenting with ophthalmoparesis from cranial nerve palsy. Neurology 2020. Doi: 10.1212/WNL.0000000000009700

26 Toscano G, Palmerini F, Ravaglia S, et al. Guillain-Barré Syndrome associated with SARS-CoV-2. N Engl J Med 2020. Doi: 10.1056/NEJMc2009191
27 Zhao C, Cao Z, Yang J, Xian K, Li X. Image feature correspondence selection: a comparative study and a new contribution. IEEE Trans Image Process 2020;4422(20):2-3

28 Sedaghat Z, Karimi N. Guillain Barre syndrome associated with COVID-19 infection: A case report. J Clin Neurosci 2020;76:233-235 